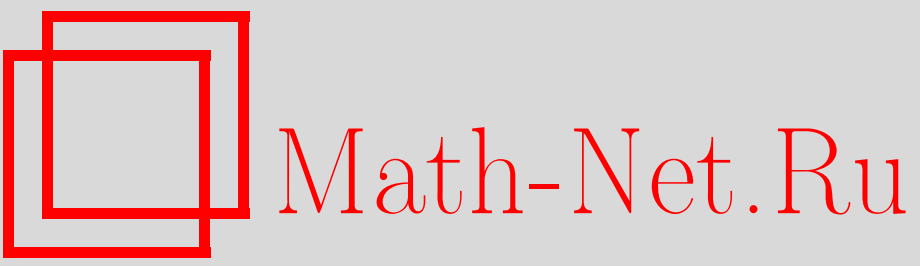

A. V. Seliverstov, Cubic forms without monomials in two variables, Vestn. Udmurtsk. Univ. Mat. Mekh. Komp. Nauki, 2015, Volume 25, Issue 1, 71-77

Use of the all-Russian mathematical portal Math-Net.Ru implies that you have read and agreed to these terms of use

http: //www. mathnet.ru/eng/agreement

Download details:

IP : 3.85 .73 .92

April 26, 2023, 18:23:11 


\title{
УДК 512.647
}

\section{(C) А.В. Селиверстов}

\section{КУБИЧЕСКИЕ ФОРМЫ БЕЗ МОНОМОВ ОТ ДВУХ ПЕРЕМЕННЫХ ${ }^{1}$}

\begin{abstract}
Доказано, что общая кубическая форма над полем комплексных чисел преобразуется к виду без мономов от ровно двух переменных каждый посредством невырожденной линейной замены координат. Если коэффициенты при мономах от одной переменной равны единице, а остальные коэффициенты принадлежат достаточно маленькому полидиску около нуля, то преобразование может быть аппроксимировано с помощью итерационного алгоритма. При этих ограничениях тот же результат справедлив над полем вещественных чисел. Этот результат обобщает теорему Леви-Деспланка о матрицах со строгим диагональным преобладанием. Нами подробно рассмотрены свойства приводимых кубических форм. Так нами доказано существование приводимой вещественной кубической формы, которая не эквивалентна никакой форме со всеми мономами от ровно одной переменной и без мономов от ровно двух переменных каждый. Предложено достаточное условие существования особой точки на проективной кубической гиперповерхности. Обсуждается вычислительная сложность распознавания особых точек.
\end{abstract}

Ключевые слова: кубическая форма, линейное преобразование, особая точка.

\section{Введение}

Кривые и поверхности третьей степени широко применяются для аппроксимации и интерполяции. Хорошо известны свойства плоских кривых Безье. Известна интерполяция кусочнокубическими функциями на плоскости, для которой оценка погрешности зависит только от диаметра разбиения, но не зависит от углов триангуляции [1]. Аналогичные результаты для многочленов шестой степени получены в [2]. Сплайны для функций многих переменных рассмотрены в [3]. Напомним, что с формой связан гиперграф, у которого вершины соответствуют переменным, а ребра - мономам. В случае квадратичных форм получается граф с петлями [4, определение 6.2.11]. Для кубических форм можно определить собственные векторы и собственные значения [5], однако их свойства существенно отличаются от свойств их аналогов для квадратичных форм. Число различных собственных значений может существенно превышать число переменных, что затрудняет их практическое использование в высоких размерностях [6]. Несмотря на вычислительные трудности, возможно применение спектральных методов в теории гиперграфов [7] и для решения прикладных задач в биоинформатике, в частности для анализа генных сетей [8]. Отметим, что гиперграфы естественно возникают при изучении сложных регуляторных систем с одновременным воздействием большого числа факторов.

В общем случае кубическая форма может быть записана в виде

$$
f=\sum_{k=0}^{n} \alpha_{k} x_{k}^{3}-3 \sum_{j \neq k} \beta_{j k} x_{j}^{2} x_{k}-3 \sum_{0 \leqslant i<j<k \leqslant n} \gamma_{i j k} x_{i} x_{j} x_{k} .
$$

Очевидно, если некоторый коэффициент формы отличен от нуля, то невырожденным преобразованием координат его легко сделать равным 1. Можно ли невырожденным линейным преобразованием координат обратить в нуль все коэффициенты $\beta_{j k}=0$ при мономах от двух переменных? Это преобразование служит некоторым аналогом диагонализации симметричных матриц и позволяет судить об инвариантных свойствах форм. С другой стороны, поскольку размерность ортогональной группы вдвое меньше числа коэффициентов $\beta_{j k}$, в общем случае такое преобразование не может быть ортогональным.

\footnotetext{
${ }^{1}$ Работа выполнена при частичной финансовой поддержке РФФИ (проект 13-04-40196-Н КОМФИ).
} 
Векторы обозначаются полужирными буквами $\mathbf{x}, \mathbf{x}^{(m)}, \mathbf{y}$. Матрицы обозначаются буквами $L$ или $L^{(m)}$. Верхний индекс в круглых скобках обозначает номер вектора или матрицы, нижний индекс - номер координаты или матричного элемента. Нумерация координат начинается с нуля. Через $\operatorname{diag}\left(\alpha_{0}, \ldots, \alpha_{n}\right)$ обозначена диагональная матрица порядка $n+1$ с элементами $\alpha_{0}, \ldots, \alpha_{n}$ на главной диагонали.

Определение 1. Две формы $f(\mathbf{x})$ и $g(\mathbf{x})$ от равного числа переменных эквивалентны над полем вещественных чисел $\mathbb{R}$ или комплексных чисел $\mathbb{C}$, если существует такой невырожденный линейный оператор $L$ в линейном пространстве над этим полем, что $f(\mathbf{x})=g(L \mathbf{x})$.

Над полем комплексных чисел кубическая форма от трех переменных, определяющая гладкую проективную кривую, эквивалентна форме без мономов от двух переменных $[9$, глава $1, \S 4]$ или [10]. Для кубической формы от четырех переменных, определяющей гладкую проективную поверхность, эквивалентность форме без мономов от двух переменных доказана в $[11,12]$ алгебраическими методами.

Определение 2 (см. [4, определение 6.1.9]). Матрица $L$ называется матрицей со строгим диагональным преобладанием, если для каждого индекса $0 \leqslant k \leqslant n$ элемент на главной диагонали удовлетворяет строгому неравенству

$$
\left|L_{k k}\right|>\sum_{j \neq k}\left|L_{k j}\right|
$$

\section{§1. Результаты}

Теорема 1. Для каждого натурального числа $n$ существует такое маленькое положительное число $0<\delta<1$, что при любых значениях коэффициентов, удовлетворяющих неравенствам $\left|\beta_{j k}\right|<\delta$ при $j \neq k u\left|\gamma_{i j k}\right|<\delta$ при $i<j<k$, кубическая форма

$$
\sum_{k=0}^{n} x_{k}^{3}-3 \sum_{j \neq k} \beta_{j k} x_{j}^{2} x_{k}-3 \sum_{0 \leqslant i<j<k \leqslant n} \gamma_{i j k} x_{i} x_{j} x_{k}
$$

эквивалентна форме вида

$$
\sum_{k=0}^{n} y_{k}^{3}-3 \sum_{0 \leqslant i<j<k \leqslant n} \lambda_{i j k} y_{i} y_{j} y_{k} .
$$

При этом если все коэффициенты $\beta_{j k} u \gamma_{i j k}$ вещественные, то все коэффициенты $\lambda_{i j k}$ тоже будут вещественными.

Д о к а з а т е л ь с т в о. Будем строить последовательность невырожденных линейных преобразований и последовательность кубических форм вида (1) с коэффициентами $\alpha_{k}^{(m)}, \beta_{j k}^{(m)}$ и $\gamma_{i j k}^{(m)}$ на шаге $m$. Здесь $\mathbf{x}^{(0)}=\mathbf{x}, \alpha_{k}^{(0)}=1, \beta_{j k}^{(0)}=\beta_{j k}$ и $\gamma_{i j k}^{(0)}=\gamma_{i j k}$. Определим подстановки координат на шаге $m$ формулой

$$
x_{j}^{(m)}=x_{j}^{(m+1)}+\frac{1}{\alpha_{j}^{(m)}} \sum_{k \neq j} \beta_{j k}^{(m)} x_{k}^{(m+1)} .
$$

Непосредственная проверка показывает, что при достаточно маленьком $\delta$ на каждом ненулевом шаге $\alpha_{k}^{(m)}=\alpha_{k}^{(m-1)}+O\left(\delta^{m}\right), \beta_{j k}^{(m)}=O\left(\delta^{m}\right)$ и $\gamma_{i j k}^{(m)}=\gamma_{i j k}^{(m-1)}+O\left(\delta^{m}\right)$. Поэтому существуют пределы $\lim _{m \rightarrow \infty} \alpha_{k}^{(m)}=\rho_{k}=1+O(\delta) \neq 0, \lim _{m \rightarrow \infty} \beta_{j k}^{(m)}=0$ и $\lim _{m \rightarrow \infty} \gamma_{i j k}^{(m)}=\mu_{i j k}$. Полагаем

$$
\lambda_{i j k}=\frac{\mu_{i j k}}{\sqrt[3]{\rho_{i}} \sqrt[3]{\rho_{j}} \sqrt[3]{\rho_{k}}}
$$


Выбор значения корня однозначен над полем вещественных чисел, но над полем комплексных чисел возможно несколько вариантов ответа. При этом для каждого значения индекса $k$ значение корня $\sqrt[3]{\rho_{k}}$ должно быть фиксировано.

Обозначим через $L^{(m)}$ матрицу линейного преобразования координат на шаге $m$. На главной диагонали этой матрицы стоят единицы, а модули других элементов ограничены сверху оценкой $O\left(\delta^{m}\right)$. Поэтому при достаточно маленьких $\delta$ существует предел произведений

$$
L=\lim _{m \rightarrow \infty} L^{(m)} \cdots L^{(0)} .
$$

Более того, $L$ - матрица со строгим диагональным преобладанием. В силу теоремы ЛевиДеспланка она невырожденная [4, следствие 5.6.17 или теорема 6.1.10]. Новые координаты выражаются через исходные по формуле

$$
\mathbf{y}=\operatorname{diag}\left(\sqrt[3]{\rho_{0}}, \ldots, \sqrt[3]{\rho_{n}}\right) L \mathbf{x}
$$

для фиксированных значений кубических корней.

Следствие 1. Общая кубическал форма над полем комплексных чисел эквивалентна форме без мономов от ровно двух переменных.

Д о к а з а т е л ь с т в о. Без ограничения общности достаточно рассмотреть семейство форм того же вида, что и в посылке теоремы 1. Существование искомого невырожденного линейного преобразования выразимо предложением языка теории поля с параметрами, равными коэффициентам исходной кубической формы. Поскольку элементарная теория поля комплексных чисел допускает элиминацию кванторов [13], это условие эквивалентно конъюнкции элементарных дизъюнкций равенств вида $p_{\ell}=0$ и их отрицаний вида $p_{\ell} \neq 0$, где $p_{\ell}-$ некоторый многочлен положительной степени от параметров. Здесь индекс $\ell$ пробегает конечное множество. Возможны два случая.

Первый случай. Если некоторая элементарная дизъюнкция содержит только равенства, условие может быть выполнено только при равенстве нулю произведения некоторых многочленов $p_{\ell}$. По теореме 1 это произведение равно нулю на некотором достаточно маленьком полидиске в пространстве параметров. Следовательно, один из многочленов $p_{\ell}$ тождественно равен нулю. Это противоречит нашему предположению об общности кубической формы, то есть этот случай невозможен.

Второй случай. Если каждая элементарная дизъюнкция содержит неравенство, условие выполнено на почти всех наборах параметров за исключением некоторого множества наборов параметров, на каждом из которых обращается в нуль хотя бы один из многочленов $p_{\ell}$. Это и требовалось доказать.

Теорема 2. Для любого $n \geqslant 2$ если форма

$$
f=\sum_{k=0}^{n} x_{k}^{3}-3 \sum_{0 \leqslant i<j<k \leqslant n} \lambda_{i j k} x_{i} x_{j} x_{k}
$$

приводимая, то для всех индексов $0 \leqslant i<j<k \leqslant n$ выполнено $\lambda_{i j k}^{3}=1$. Более того, форма $f$ равна произведению форм

$$
f=\left(\sum_{i=0}^{n} \alpha_{i} x_{i}\right)\left(\sum_{k=0}^{n} \alpha_{k}^{2} x_{k}^{2}-\sum_{0 \leqslant j<k \leqslant n} \alpha_{j} \alpha_{k} x_{j} x_{k}\right),
$$

где для любого индекса $k$ выполнено равенство $\alpha_{k}^{3}=1$. 
Д о к а з а т е л ь с т в о. Приводимость формы означает, что существует разложение

$$
f=\left(\sum_{i=0}^{n} \alpha_{i} x_{i}\right)\left(\sum_{j=0}^{n} \sum_{k=0}^{n} \beta_{j k} x_{j} x_{k}\right),
$$

где $\beta_{j k}=\beta_{k j}$. При этом для каждого индекса $k$ выполнено $\alpha_{k} \beta_{k k}=1$, следовательно, $\alpha_{k} \neq 0$ и $\beta_{k k} \neq 0$. Для любых $j \neq k$ выполнено $2 \alpha_{k} \beta_{j k}+\alpha_{j} \beta_{k k}=0$. Умножая последнее равенство на $\alpha_{k}$ и используя равенство $\alpha_{k} \beta_{k k}=1$, получим $2 \alpha_{k}^{2} \beta_{j k}+\alpha_{j}=0$. Поскольку $\alpha_{k} \neq 0$, получим $\beta_{j k}=-\frac{\alpha_{j}}{2 \alpha_{k}^{2}}$. Поскольку $\beta_{j k}=\beta_{k j}$, получаем для любых двух индексов $j$ и $k$ равенство $\alpha_{k}^{3}=\alpha_{j}^{3}$. Без ограничения общности можно выбрать $\alpha_{0}=1$. Тогда для каждого индекса $k$ выполнены равенства $\alpha_{k}^{3}=\beta_{k k}^{3}=1$ и $\beta_{k k}=\alpha_{k}^{2}$. Также для любых двух индексов $j \neq k$ выполнено равенство $2 \beta_{j k}=-\alpha_{j} \alpha_{k}$.

Замечание 1. При $n=1$ множество индексов $0 \leqslant i<j<k \leqslant n$ пустое. Единственная кубическая форма рассматриваемого вида $f=x_{0}^{3}+x_{1}^{3}=\left(x_{0}+x_{1}\right)\left(x_{0}^{2}-x_{0} x_{1}+x_{1}^{2}\right)$ приводимая, а множители такие, как в теореме 2.

Замечание 2. При $n=2$ форма $f$ в теореме 2 равна произведению трех линейных форм $[9$, 10]. Обозначим через $\varepsilon$ корень многочлена $\varepsilon^{2}+\varepsilon+1$. Тогда

$$
x_{0}^{3}+x_{1}^{3}+x_{2}^{3}-3 x_{0} x_{1} x_{2}=\left(x_{0}+\varepsilon x_{1}+\varepsilon^{2} x_{2}\right)\left(x_{0}+\varepsilon^{2} x_{1}+\varepsilon x_{2}\right)\left(x_{0}+x_{1}+x_{2}\right) .
$$

Следствие 2. Для любого $n \geqslant 2$ существует вещественная кубическая форма от $n+1$ переменной, не эквивалентнал над полем комплексных чисел никакой форме вида

$$
\sum_{k=0}^{n} x_{k}^{3}-3 \sum_{0 \leqslant i<j<k \leqslant n} \lambda_{i j k} x_{i} x_{j} x_{k} .
$$

Д о к а з а т е л ь с т в о. Согласно теореме 2 над полем вещественных чисел существует только одна приводимая кубическая форма указанного вида. При $n \geqslant 2$ все ее коэффициенты $\lambda_{i j k}=1$. Следовательно, достаточно указать две неэквивалентные друг другу приводимые вещественные формы. Одна из них - произведение трех линейных форм, а другая - произведение линейной и неприводимой квадратичной формы.

\section{§ 2. Обсуждение}

Доказано, что почти любая кубическая форма над полем комплексных чисел эквивалентна форме специального вида. Более того, в доказательстве теоремы 1 при ограничении на модули коэффициентов получен итерационный алгоритм вычисления этой формы. Это может быть полезно для исследования кубической гиперповерхности (кубики) в проективном пространстве. Некоторые возможные приложения обсуждаются в работе [14]. В частности, следствие 1 приводит к интересному достаточному условию существования особой точки. Действительно, проективная гиперповерхность, заданная формой

$$
f=\sum_{k=0}^{n-1} \rho_{k} x_{k}^{3}-3 \sum_{0 \leqslant i<j<k \leqslant n} \lambda_{i j k} x_{i} x_{j} x_{k},
$$

то есть при $\rho_{n}=0$, содержит особую точку с однородными координатами $(0: \ldots: 0: 1)$. Но следствие 2 говорит, что в отличие от квадрик мы не получаем достаточного условия гладкости кубики. Более того, задача распознавания особых кубик является алгоритмически трудной. Напомним, что дискриминант формы равен нулю, если соответствующая проективная гиперповерхность имеет особую точку [15]. Трудность распознавания особых гиперповерхностей связана с тем, что для формы степени $d$ от переменных $x_{0}, \ldots, x_{n}$ дискриминант имеет степень 
$(n+1)(d-1)^{n}$. Дискриминант квадратичной формы пропорционален определителю ее матрицы и имеет степень $n+1$. Однако степень дискриминанта кубической формы очень быстро растет с увеличением числа переменных. Непосредственная проверка существования нетривиального общего нуля у всех частных производных общей кубической формы также требует времени, экспоненциально зависимого от числа переменных $[13,16]$.

Доказательство теоремы 1 содержит описание алгоритма вычисления разложения Варинга для бинарных кубических форм при соответствующих ограничениях на коэффициенты. В общем случае проверка равенства ранга 3-тензора данному числу [17] и вычисление разложения Варинга для кубических форм [18] - это алгоритмически трудные задачи. Отметим, что произведение трех переменных равно сумме четырех кубов линейных форм

$$
24 x_{0} x_{1} x_{2}=\left(x_{0}+x_{1}+x_{2}\right)^{3}+\left(x_{0}-x_{1}-x_{2}\right)^{3}+\left(x_{1}-x_{0}-x_{2}\right)^{3}+\left(x_{2}-x_{0}-x_{1}\right)^{3},
$$

но не равно никакой сумме меньшего числа кубов [19].

Методы доказательства теоремы 1 и следствия 1 можно перенести на формы других степеней. Для квадратичных форм аналогом теоремы 1 служит теорема Леви-Деспланка [4, следствие 5.6.17 или теорема 6.1.10]. Следствие 1 аналогично приведению квадратичной формы к каноническому виду. При этом для квадратичных форм получены более точные результаты. В частности, канонический вид квадратичной формы всегда позволяет определить гладкость проективной квадрики.

\section{СПИСОК ЛИТЕРАТУРЫ}

1. Латыпова Н.В. Независимость оценок погрешности интерполяции кубическими многочленами от углов треугольника // Труды Института математики и механики УрО РАН. 2011. Т. 17. № 3. C. $233-241$.

2. Латыпова Н.В. Погрешность интерполяции многочленами шестой степени на треугольнике // Вестник Удмуртского университета. Математика. Механика. Компьютерные науки. 2013. Вып. 4. C. $79-87$.

3. Родионов В.И. О применении специальных многомерных сплайнов произвольной степени в численном анализе // Вестник Удмуртского университета. Математика. Механика. Компьютерные науки. 2010. Вып. 4. С. 146-153.

4. Хорн Р., Джонсон Ч. Матричный анализ. М.: Мир, 1989. 655 с.

5. Hu S., Qi L. The E-eigenvectors of tensors // Linear and Multilinear Algebra. 2014. Vol. 62. № 10. P. 1388-1402.

6. Cartwright D., Sturmfels B. The number of eigenvalues of a tensor // Linear Algebra and its Applications. 2013. Vol. 438. № 2. P. 942-952.

7. Shao J., Qi L., Hu S. Some new trace formulas of tensors with applications in spectral hypergraph theory // Linear and Multilinear Algebra. 2015. Vol. 63. № 5. P. 971-992.

8. Евдокимов А.А., Кочемазов С.Е., Отпущенников И.В., Семёнов А.А. Исследование дискретноавтоматных моделей генных сетей нерегулярной структуры методами символьных вычислений // Дискретный анализ и исследование операций. 2014. Т. 21. № 3. С. 25-40.

9. Прасолов В.В., Соловьев Ю.П. Эллиптические функции и алгебраические уравнения. М.: Факториал, 1997. $288 \mathrm{c}$.

10. Artebani M., Dolgachev I. The Hesse pencil of plane cubic curves // L'Enseignement Mathématique. 2009. Vol. 55. № 3/4. P. 235-273.

11. Emch A. On a new normal form of the general cubic surface // American Journal of Mathematics. 1931. Vol. 53. № 4. P. 902-910.

12. Emch A. Properties of the cubic surface derived from a new normal form // American Journal of Mathematics. 1939. Vol. 61. № 1. P. 115-122.

13. Григорьев Д.Ю. Сложность разрешения теории первого порядка алгебраически замкнутых полей // Известия АН СССР. Серия математическая. 1986. Т. 50. № 5. С. 1106-1120.

14. Гершгорин Р.А., Рубанов Л.И., Селиверстов А.В. Легко вычислимые инварианты для распознавания гиперповерхности // Информационные процессы. 2014. Т. 14. № 4. С. 365-369.

15. Морозов А.Ю., Шакиров Ш.Р. Новые и старые результаты в теории результантов // Теоретическая и математическая физика. 2010. Т. 163. № 2. С. 222-257. 
16. Chistov A.L. An improvement of the complexity bound for solving systems of polynomial equations // Записки научных семинаров ПОМИ. 2011. Т. 390. С. 299-306.

17. Håstad J. Tensorrank is NP-complete// Journal of Algorithms. 1990. Vol. 11. P. 644-654.

18. Oeding L., Ottaviani G. Eigenvectors of tensors and algorithms for Waring decomposition // Journal of Symbolic Computation. 2013. Vol. 54. P. 9-35.

19. Гашков С.Б., Шавгулидзе Е.Т. О представлении произведений в виде суммы степеней линейных форм // Вестник Московского университета. Серия 1: Математика. Механика. 2014. № 2. С. 9-14.

Поступила в редакцию 16.01 .2015

Селиверстов Александр Владиславович, к. ф.-м.н., ведущий научный сотрудник, лаборатория 6, Институт проблем передачи информации им. А. А. Харкевича Российской академии наук, 127051, Россия, г. Москва, Большой Каретный пер., 19, стр. 1.

E-mail: slvstv@iitp.ru

\section{A. V. Seliverstov}

\section{Cubic forms without monomials in two variables}

Keywords: cubic form, linear transformation, singular point.

MSC: 15A69, 14J70, 32S25

It is proved that a general cubic form over the field of complex numbers can be transformed into a form without monomials of exactly two variables by means of a non-degenerate linear transformation of coordinates. If the coefficients of monomials in only one variable are equal to one, and the remaining coefficients belong to sufficiently small polydisc near zero, then the transformation can be approximated by iterative algorithm. Under these restrictions the same result holds over the reals. This result generalizes the Levy-Desplanques theorem on strictly diagonally dominant matrices. We discuss in detail the properties of reducible cubic forms. So we prove the existence of a reducible real cubic form that is not equivalent to any form with all monomials in only one variable and without any monomials in exactly two variables. We suggest a sufficient condition for the existence of a singular point on a projective cubic hypersurface. The computational complexity of singular points recognition is discussed.

\section{REFERENCES}

1. Latypova N.V. Independence of error estimates of interpolation by cubic polynomials from the angles of a triangle, Tr. Inst. Mat. Mekh. Ural. Otd. Ross. Akad. Nauk, 2011, vol. 17, no. 3, pp. $233-241$ (in Russian).

2. Latypova N.V. Error of interpolation by sixth-degree polynomials on a triangle, Vestn. Udmurt. Univ. Mat. Mekh. Komp'yut. Nauki, 2013, no. 4, pp. 79-87 (in Russian).

3. Rodionov V.I. On application of special multivariate splines of any degree in the numerical analysis, Vestn. Udmurt. Univ. Mat. Mekh. Komp'yut. Nauki, 2010, no. 4, pp. 146-153 (in Russian).

4. Horn R.A., Johnson C.R. Matrix analysis, Cambridge University Press, 1986, 575 p. Translated under the title Matrichnyi analiz, Moscow: Mir, 1989, 655 p.

5. Hu S., Qi L. The E-eigenvectors of tensors, Linear and Multilinear Algebra, 2014, vol. 62, no. 10, pp. 1388-1402.

DOI: $10.1080 / 03081087.2013 .828721$

6. Cartwright D., Sturmfels B. The number of eigenvalues of a tensor, Linear Algebra Appl., 2013, vol. 438, no. 2, pp. 942-952.

DOI: $10.1016 /$ j.laa.2011.05.040

7. Shao J., Qi L., Hu S. Some new trace formulas of tensors with applications in spectral hypergraph theory, Linear and Multilinear Algebra, 2015, vol. 63, no. 5, pp. 971-992.

DOI: $10.1080 / 03081087.2014 .910208$ 
8. Evdokimov A.A., Kochemazov S.E., Otpushchennikov I.V., Semenov A.A. Study of discrete automaton models of gene networks of nonregular structure using symbolic calculations, Journal of Applied and Industrial Mathematics, 2014, vol. 8, no. 3, pp. 307-316.

DOI: $10.1134 /$ S1990478914030028

9. Prasolov V., Solovyev Yu. Elliptic functions and elliptic integrals, Translations of Mathematical Monographs, Book 170, American Mathematical Society, 1997, 185 p. Original Russian text published in Prasolov V.V., Solovyev Yu.P. Ellipticheskie funktsii $i$ algebraicheskie uravneniya, Moscow: Factorial, 1997, 288 p.

10. Artebani M., Dolgachev I. The Hesse pencil of plane cubic curves, Enseign. Math., 2009, vol. 55, no. 3/4, pp. 235-273.

DOI: $10.4171 / \mathrm{LEM} / 55-3-3$

11. Emch A. On a new normal form of the general cubic surface, Amer. J. Math., 1931, vol. 53, no. 4, pp. 902-910.

12. Emch A. Properties of the cubic surface derived from a new normal form, Amer. J. Math., 1939, vol. 61, no. 1, pp. 115-122.

13. Grigor'ev D.Yu. The complexity of the decision problem for the first order theory of algebraically closed fields, Mathematics of the USSR. Izvestiya, 1987, vol. 29, no. 2, pp. 459-475.

DOI: 10.1070/IM1987v029n02ABEH000979

14. Gershgorin R.A., Rubanov L.I., Seliverstov A.V. Easy computable invariants for hypersurface recognition, Information Processes, 2014, vol. 14, no. 4, pp. 365-369 (in Russian). http://www.jip.ru/2014/365-369-2014.pdf

15. Morozov A.Yu., Shakirov Sh.R. New and old results in resultant theory, Theoret. and Math. Phys., 2010, vol. 163 , no. 2, pp. 587-617.

DOI: $10.1007 / \mathrm{s} 11232-010-0044-0$

16. Chistov A.L. An improvement of the complexity bound for solving systems of polynomial equations, J. Math. Sci. (New York), 2012, vol. 181, no. 6, pp. 921-924.

DOI: $10.1007 / \mathrm{s} 10958-012-0724-4$

17. Håstad J. Tensorrank is NP-complete, J. Algorithms, 1990, vol. 11, pp. 644-654.

DOI: $10.1016 / 0196-6774(90) 90014-6$

18. Oeding L., Ottaviani G. Eigenvectors of tensors and algorithms for Waring decomposition, J. Symbolic Comput., 2013, vol. 54, pp. 9-35.

DOI: $10.1016 /$ j.jsc.2012.11.005

19. Gashkov S.B., Shavgulidze E.T. Representation of monomials as a sum of powers of linear forms, Moscow Univ. Math. Bull., 2014, vol. 69, no. 2, pp. 51-55.

DOI: $10.3103 / \mathrm{S} 0027132214020028$

Received 16.01.2015

Seliverstov Alexandr Vladislavovich, Candidate of Physics and Mathematics, Leading Researcher, Laboratory 6 , Institute for Information Transmission Problems of the Russian Academy of Sciences (Kharkevich Institute), Bol'shoi Karetnyi per., 19, build. 1, Moscow, 127051, Russia.

E-mail: slvstv@iitp.ru 